\title{
Fundamentals of Gamma-Ray Measurements and Radiometric Analyses
}

by

R. C. Hochel

Westinghouse Savannah River Company

Aiken, South Carolina 29808

\author{
A paper proposed for presentation at a \\ Traveling Lecturer Seminar at the Citadel \\ Charleston, S. C. \\ February 20, 1990
}

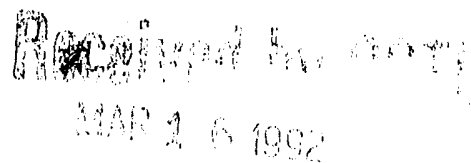

\section{DISCLAIMER}

\begin{abstract}
This report was prepared as an account of work sponsored by an agency of the United States Government. Neither the United States Government nor any agency thereof, nor any of their employees, makes any warranty, express or implied, or assumes any legal liability or responsibility for the aceuracy, completeness, or usefulness of any information, apparatus, product, or process disclosed, or represents that its use would not infringe privately owned riglits. Reference herein to any specific commercial product, process, or service by trade name, trademark, manufacturer, or otherwise does not necessarily constitute or imply its endorsement, recommendation, or favoring by the United States Government or any agency thereof. The views and opinions of authors expressed herein do not necessarily state or reflect those of the United States Government or any agency thereof.
\end{abstract}

This paper was prepared in connection with work done under Contract No. DE-AC09-88SR18035 with the U.S. Department of Energy. By acceptance of this paper, the publisher and/or recipient acknowledges the U.S.

Government's right to retain a nonexclusive, royalty-free license in and to any copyright covering this paper, along with the right to reproduce and to authorize othors to reproduce all or part of the copyrighted paper.

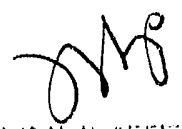




\section{FUNDAMENTALS OF GAMMA-RAY MEASUREMENTS AND RADIOMETRIC ANALYSES - A TRAVELING LECTURE SEMINAR (Unclassified)}

By

R. C. Hochel

Analytical Development Section

Savannah River Laboratory

To be pressented at The Citadel in Charleston, SC 2/20/90

Thank you for inviting me to The Citadel and the opportunity to talk to you about gamma-ray measurements and radiometric analy'ses, a subject near and dear to my heart since coming to the Savannah. River Site and Laboratory nearly 17 years ago. Professor Abelrnan tells me that you haven't much in the way of nuclear physics or laboratory work with radiation measurements and their uses, so I will try to introduce you to some of the fundamentals. Hopefully some of you will find it interesting enough to pursue in your future studies, as today there is real shortage of nuclear chemists and physicists in this country.

Slide 1 Perhaps the best place to start is with the radioactive decay law which is the basis of all radiometric analyses. It simply states the rate of decay, $-\mathrm{dN} / \mathrm{dt}$, of any radioactive nucleus is proportional to the number of nuclei, $\mathrm{N}$, present at some time, $t$. The decay constant, $\lambda$, is given by the natural $\log$ of 2 over the half-life, $t_{1 / 2}$. Tris differential equation can be easily integrated to give an expression for the number of nuclei remaining at any time, given the number at time zero. If only one radioactive species is present, the decay rate can be measured directly to give the number of radionuclei present in the sample. However, if more than one species is present, a chemical separation or a spectroscopy measurement, capable of distinguishing between the decaying species, must be performed. It

Derivative Classifier_Eevgenlem 
should be pointed out that such analyses are isotope specific because the measured radiation originates in the nucleus and not the chemical atom. Consequently, we can easily tell if say a cesium sample contains Cs-134 or Cs-137, but we would need to know all the cesium isotopes, and sum them up to determine the total cesium content.

Slide 2 There are four primary modes of radioactive decay. All can be measured using various types of detectors and are the basis of many analytical techniques and much of what we know about the nucleus and its structure. Alpha particle emission occurs mostly in heavy nuclei of atomic number, $\mathrm{Z}$, greater than 82 like $\mathrm{Po}, \mathrm{Ra}, \mathrm{Th}$, and $\mathrm{U}$, etc. The alpha particle is a helium nucleus of atomic mass $4,(2$ protons and 2 neutrons) and is usually emitted in discrete energies between 3.5-6.0 MeV. Because of its +2 charge, it looses energy in matter rapidly, and must be measured with a thin window detector and often in vacuum. Beta particles are simply electrons. They are emitted from the nucleus with a distribution of energies ranging from $0-3 \mathrm{MeV}$. The maximum energy is a specific property of the decaying isotope. Again, being charged particles, they loose energy fairly rapidly in matter, but have ranges greater than alpha particles Gamma-rays are photons with energies ranging from a few $\mathrm{keV}$ to 10 Mev or more. They usually follow alpha or beta decay, and depending on their energy, can have considerable range in matter. Neutrons are emitted in fission processes and also from a few of the highly excited fission product nuclei. Fission neutrons typically have energies of 1-2 $\mathrm{MeV}$. Like gamma-rays, they also have long ranges.

Slide 3 The energies involved in nuclear decay processes are much higher than anything encountered in say chemical reactions. As you can see, they are at the very top of the electromagnetic spectrum - about a million times more energetic than visible light. As a result, these particles always produce ionization, either directly or indirectly, as they pass through matter. It is this ionization which is the basis of all radiation detectors.

Slide 4 There are many types of radiation detectors, and some of the more common ones are shown here. They mostly fall into three different categories: gas-filled, scintillation, and solid-state detectors. Some of them like the Geiger/Muller, NaI, and Ge detectors, you've probably heard of before.

Slide 5 This is a schematic diagram of a gas-filled detector. Radiation, as it passes through the gas in the detector, produces 
ionization in the form of positive and negative ion pairs. If a potential is applied across the tube, the ion pairs migrate under the influence of the potential field and produce a current which can be measured. This is the principle of the ionization chamber, where the current is a direct measure of the ionization produced by the radiation. If the voltage across the tube is increased, the primary ion pairs are accelerated and produce secondary ionization which results in charge multiplication. If the voltage isn't too high, the total charge pulse remains proportional to the primary charge deposited by the radiation. Such detectors are called proportional counters, and can discriminate between alpha and beta particles because alphas produce much more ionization, as they pass through the detector, on average, than do betas. By integrating the charge on a capacitor, a voltage pulse will be produced for each event detected. In this mode of operation, the pulse output voltage is proportional to the energy deposited in the detector by the event. Because alpha and beta particles have short ranges, they deposit nearly all their energy in the detector, so its possible to measure their incident energies with such detectors. Gammas, with their longer ranges, generally will only deposit a fraction of their incident energy. If the tube voltage is increased further, any event detected will produce an avalanche of charge and a large output pulse regardless of the type of particle or its energy. In this mode of operation, the detector is called a Geiger/Muller detector.

Slide 6 This is a schematic of a scintillation detector. In this detector, ionizing events which take place in the scintillator produce light. When the light pulse strikes the photocathode, electrons are produced which are accelerated and amplified down the dynode string of the photomultiplier tube. The results in a voltage signal at the anode which is proportional to the energy of the incident particle. The stopping power of materials increase with increasing $\mathrm{Z}$ of its elemental constituents. Thus, the heavier elements like $\mathrm{Ge}, \mathrm{I}$, and $\mathrm{Pb}$ are progressively better absorbers of gamma-rays. Materials such as $\mathrm{NaI}$ and CsI are also scintillators, and thus make good gamma-ray detectors. Certain organics and plastics are also scintillators and find considerable use for alpha and beta detection. Because the photomultiplier is sensitive to light, the detector assembly must be light tight. This means that the detector window must be very thin for alphas and some betas. Consequently, liquid scintillators are often used since the sample can be mixed directly with the liquid.

Slide 7 The solid-state detector is similar to a gas detector in operation. A semiconducting material like $\mathrm{Si}$ or $\mathrm{Ge}$ is doped with $\mathrm{n}$ 
and $\mathrm{p}$ type donors, and reversed biased to form a region depleted of charge carriers. Ion pairs formed by radiation passing through the depleted region are collected to produce a voltage pulse, just as in the gas detector. However the here higher $\mathrm{Z}$ of $\mathrm{Si}$ or $\mathrm{Ge}$ make for much better detection of gamma-rays than is possible with gas detectors.

Slide 8 Gamma-rays interact with matter through secondary effects since they are uncharged. This can happen by any of three processes. In the photoelectric effect, the gamma-ray transfers all of its energy to a bound atomic electron and knocks it free. The energetic electron then causes ionization the same as a beta particle. Because of its short range, the electron is usually totally stopped in the detector, so the recorded pulse is proportional to the incident energy of the gamma-ray.

Slide 9 A second interaction process is Compton scattering. Again the gamma-ray interacts with an atomic electron, but scatters and imparts only a portion of its energy. The electron is knocked free as in the photoelectric effect, but Lne gamma-ray also exits at a lower energy. The electron will be stopped in the detector, but the scattered gamma-ray may escape. Depending on the scattering angle, $\Phi$, a range of energies from almost zero $(\Phi=0)$ to some maximum $(\Phi=180)$ may be imparted to the electron.

Slide 10 The third method of interaction is pair production. Here the gamma-ray in passing near a nucleus disappears and creates a positron-electron pair. Because the rest mass energy of the pair is $1.022 \mathrm{MeV}$, this process is only possible if the gamma-ray energy exceeds this value. Any excess energy of the gamma-ray is converted to kinetic energy of the pair. Again, the pair the pair will usually be stopped in the detector, and the total incident gamma-ray energy recorded.

Slide 11 These three processes are not all equally likely. Instead, they depend on the energy of the gamma-ray and the detector material. As you can see, the photoelectric effect dominates at low energies, but falls below the slowly dropping Compton process above about $200 \mathrm{keV}$. Pair production, as I mentioned, has a threshold and only becomes important above several MeV.

Slide 12 The question of which detector to choose for a particular application depends on several factors. One of the more important 
ones is energy resolution which is shown here. As you can see, the solid-state $\mathrm{Si}(\mathrm{Li})$ detector is by far the best followed by the gas and scintillation detectors. For detection efficiency, the rankings (best worst) are: scintillation, solid-state, gas; costs are: gâs, scintillation, solid-state. Gamma-rays, because of their range, isotope specificity, and ease of measurement, are the basis of most modern radiometric analyses. And for most gamma-ray measurements, the solid-state $\mathrm{Ge}$ detector can't be beat.

Slide 13 Having talked about the different ways gamma-rays interact, let's look at a hypothetical spectrum of a monoenergetic gamma source. $A$ is the full energy peak from the photoelectric effect; $B$ is the Compton distribution; $C$ is the Compton peak for maximum energy transfer by Compton scattering; $D$ is the Compton valley due to multiple Compton interactions; $\mathrm{E}$ is the backscatter peak for gammas scattered back to the detector through 180 degrees; and $F$ and $G$ represent underlying background from other sources, cosmic events, etc.

Slide 14 Such a spectrum is typicaliy generated by this arrangement of equipment. The essentials are the detector, a detector bias supply, a preamplifier to shape the pulse and drive the signal cable, an amplifier for gain control, and a multichannel analyzer (MCA). The pulser and oscilloscope are optional devices to help in setup of the system. The multichannel analyzer consists of an analogto-digital converter (ADC), and ${ }^{n}$ memory storage and display unit. The ADC transforms the amplifier voltage signal to a digital signal which is stored in a histogram type memory. A typical MCA might have 1000-4000 channels, each capable of storing several million counts/channel.

Slide 15 This is a reproduction of an actual MCA spectrum of a sample containing 11 different radionuclei. The $x$-axis represents increasing gamma-ray energy and the $y$-axis is increasing counts per unit of energy. The peak energies directly identify the isotopes in the sample, and their intensities are a measure of the amounts present.

Slide 16 Gamma-ray and alpha or beta spectra obtained from either radioactive decay or in-beam studies on particle accelerators also provide much of what we know about nuclear structure. This shows the energy level scheme of the decay of Cs-137 to Ba-137, along with other information you would find in Nuclear Data Tables. Note there are two beta branches to $\mathrm{Ba}-137$. $6.5 \%$ of the time decay is 
to the ground state, and $93.5 \%$ the $11 / 2$ - state is populated. It's the decays to the $11 / 2$ - state that give rise to the $661.6 \mathrm{keV}$ gamma-ray associated with Cs-137 decay. Note, there is no gamma-ray emitted for beta decay to the ground state. Just as in atomic physics, nuclear states also are characterized by quantum numbers and selection rules. The decay from the $11 / 2$ - excited state to the $3 / 2+$ ground state is highly forbidden, and so the excited state has an appreciable half-life. Normally, the lifetime of excited states relative gamma emission is less than a picosecond.

Slide 17 Of course these level schemes can become quite complicated as you see here. Pu-239 decays to U-235 by alpha emission populating many states and producing well over 50 gammarays in the process.

Slide 18 This is a gamma-ray spectrum of Pu-239. Besides the many lines from Pu-239, you'll also see lines from Pu-240, Am-241, and U-2.37. When Pu-239 is produced by irradiation of U-238 in a reactor, $\mathrm{Pu}-240$ and $\mathrm{Pu}-241$ also result from successive neutron captures on the Pu-239. Pu-241 decays to Am-241 which in turn decays to U-237. This is a problem for any element having more than one radioactive isotope, and has to be considered in any measurement.

Slide 19 As was mentioned earlier, resolution is one of the deciding factors in selecting a detector for any measurement. Here we see a comparison of a NaI scintillation detector with a Ge solid-state detector. Obviously, as the number of peaks in the spectrum increases, so does the chance of significant overlap.

Slide 20 Still, poor resolution detectors, such as NaI, have advantages in detection efficiency, size, and cost which often make them the detector of choice. This is a system I designed for one of the processing areas at SRS. It uses $10 \mathrm{NaI}$ detectors and multiple ADCs to monitor various points in the process. The computer looks at each individual spectrum, extracts concentration related peak areas, and converts this digital information to an analog signal capable of driving alarms if unwanted conditions exist.

Slide 21 As I'm sure you know, there is a lot of public concern about radiation, and hardly a day goes by that we don't hear about some newly discovered contaminated area. This is because radiation is so easy to measure even in extremely small amounts, not because there is a severe environmental problem. Consider a radionuclide 
with a half-life of 100 years and an atomic weight of 100 . If we measured as little as 6 disintigations per minute for a sample, with say a Geiger/Muller counter, how much would we have? From my first slide you'll recall that the decay rate, $\mathrm{dN} / \mathrm{dt}$, is equal to the number of atoms present times the isotope's decay constant, lamda. Using the relationship that lamda is given by the natural $\log$ of 2 over the half-life, we can quickly calculate that the sample contains only about a half a billion atoms. Using the atomic weight of 100 and Avagadro's number, 6 times ten to the 23rd, we get a weight of just under ten to the -14 th grams, or about 1 gram of the radionuclide per million tons of inert material.

Slide 22 Finally, I should say something about active interrogation methods. Up until now everything I've said depends on the sample passively emitting radiation that can be measured. If the sample isn't radioactive or its radiations can'* be easily detected, sometimes it's possible to interrogate the sample to make it give some response we can measure. In X-ray fluorescence, the atoms in the sample are bombarded with $x$-rays to excite electrons and then to measure the $x$-rays emitted in deexcitation. K\&L-edge densitometry depends on the fact that there is a large concentration dependance in the mass attenuation coefficient at either the $\mathrm{K}$ or $\mathrm{L}$ absorption edges. By measuring the difference in gamma-ray absorption just below and just above the edge, concentration can be determined. In neutron activation, the sample is irradiated with neutrons to turn stable isotopes into radioactive ones. Measuring the gamma-ray activity after irradiation allows quantitative analysis of many elements simultaneously. The $\mathrm{Cf}$ shuffler is similar in that it uses neutrons to interrogate the sample. But, here the neutron source (Cf-252) is brought next to the sample and then quickly removed (shuffled). Delayed gamma-rays or neutrons from the induced fissions are measured. In some cases, gamma-rays can be used to dissociate or break-up certain nuclei. When this happens, neutrons and protons are produced which can be easily measured.

I hope that I've been able to give you some insight into nuclear radiation measurement techniques, and some of their many uses in material analyses and nuclear physics and chemistry research. If you have any questions, I'll be glad to try to answer them. Again, thank you for the opportunity to talk to you. 
Radiactive Decay Law:

$$
\begin{aligned}
-\mathrm{dN} / \mathrm{d} t & =\mathrm{N} \lambda \\
\lambda & =\ln 2 / t_{1 / 2} \\
\mathrm{~N} & =\mathrm{N}_{0} \mathrm{e}^{-\lambda t}
\end{aligned}
$$

To Distinguish Between Different Radioactive Species: Chemical separation spectroscopy

*Analyses are Isotope Specific 
- Alpha Particle Emission

$Z>82$

Energy $=3.5-6.0 \mathrm{MeV}$ (Discrete)

Beta Particle Emission

Energy $=0-3 \mathrm{MeV}$ (Distribution)

Maximum Energy is Isotope Specific

Gamma-Ray Emission

- Usually Follows Alpha/Beta Emission

Energy $=10 \mathrm{keV}-10 \mathrm{MeV}$ (Discrete)

Neutron Emission

Usually Follows Fission

Energy $=1-2 \mathrm{MeV}$ (Distribution) 


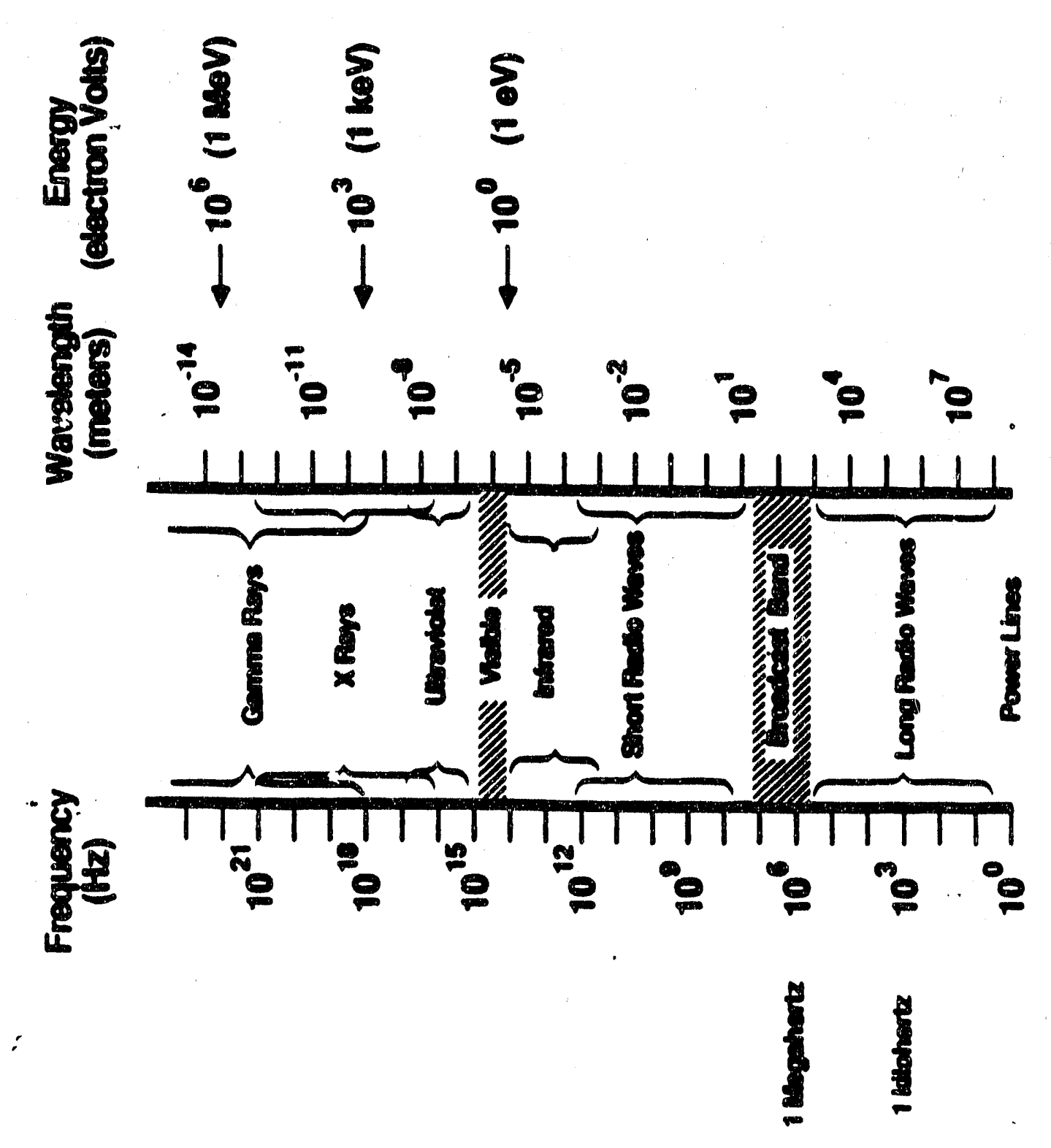




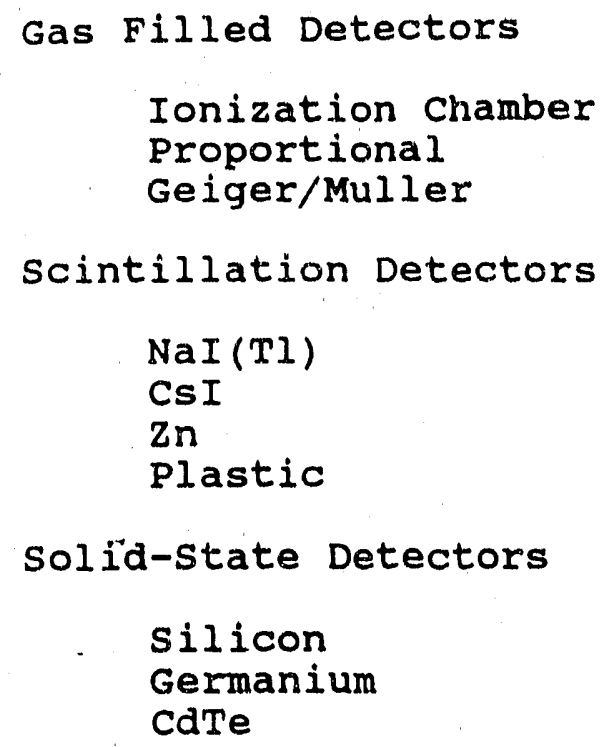




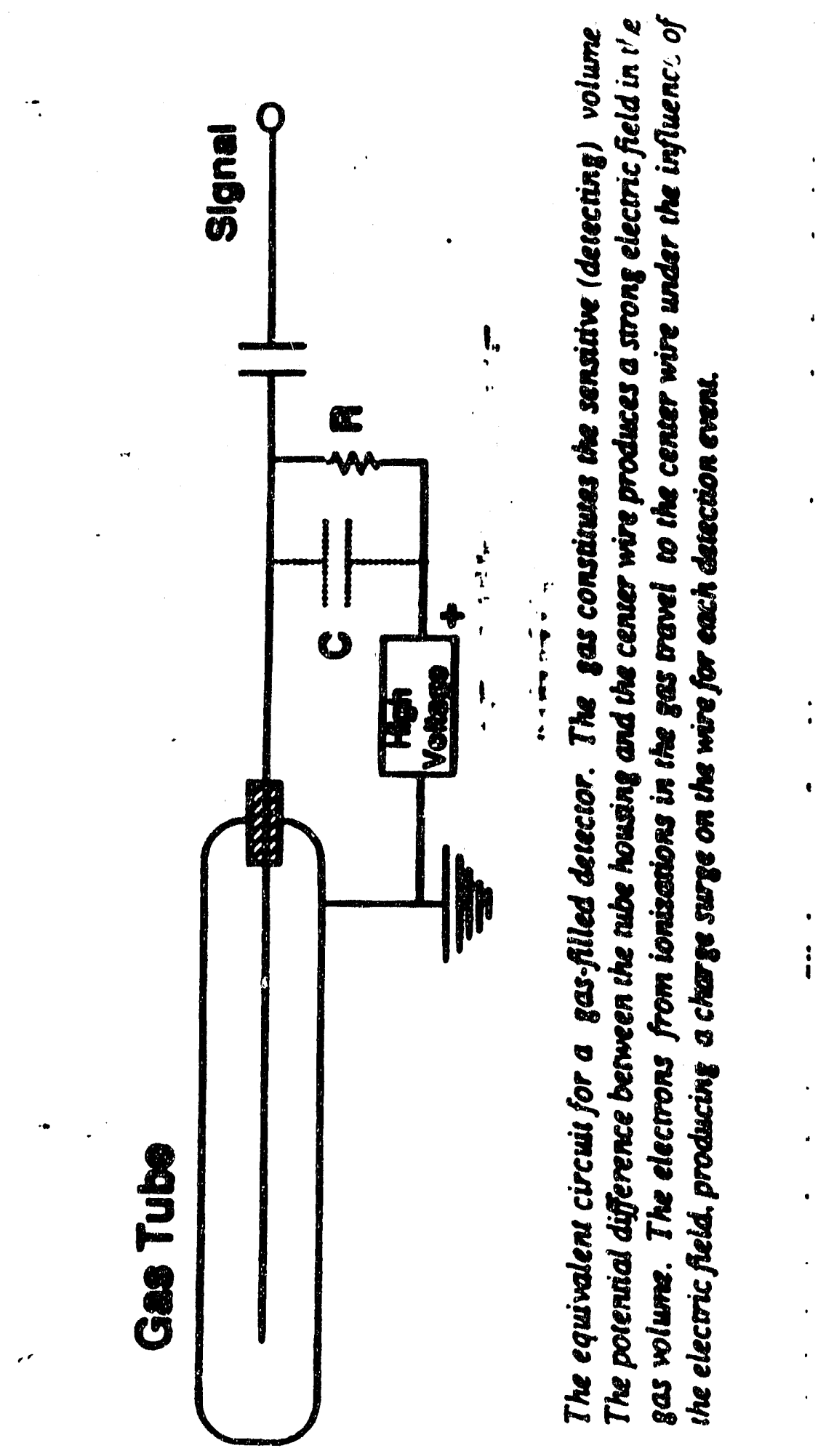




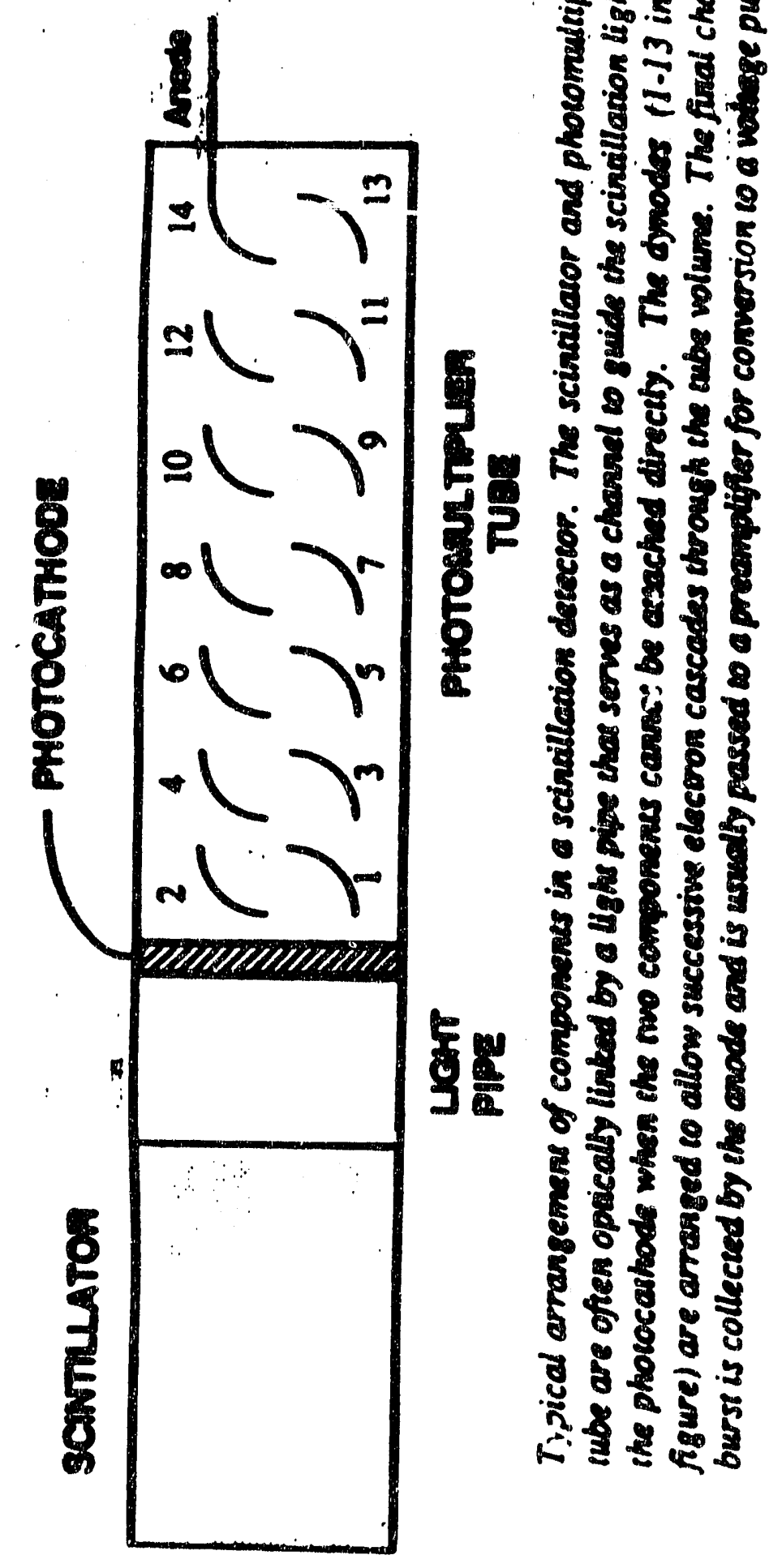




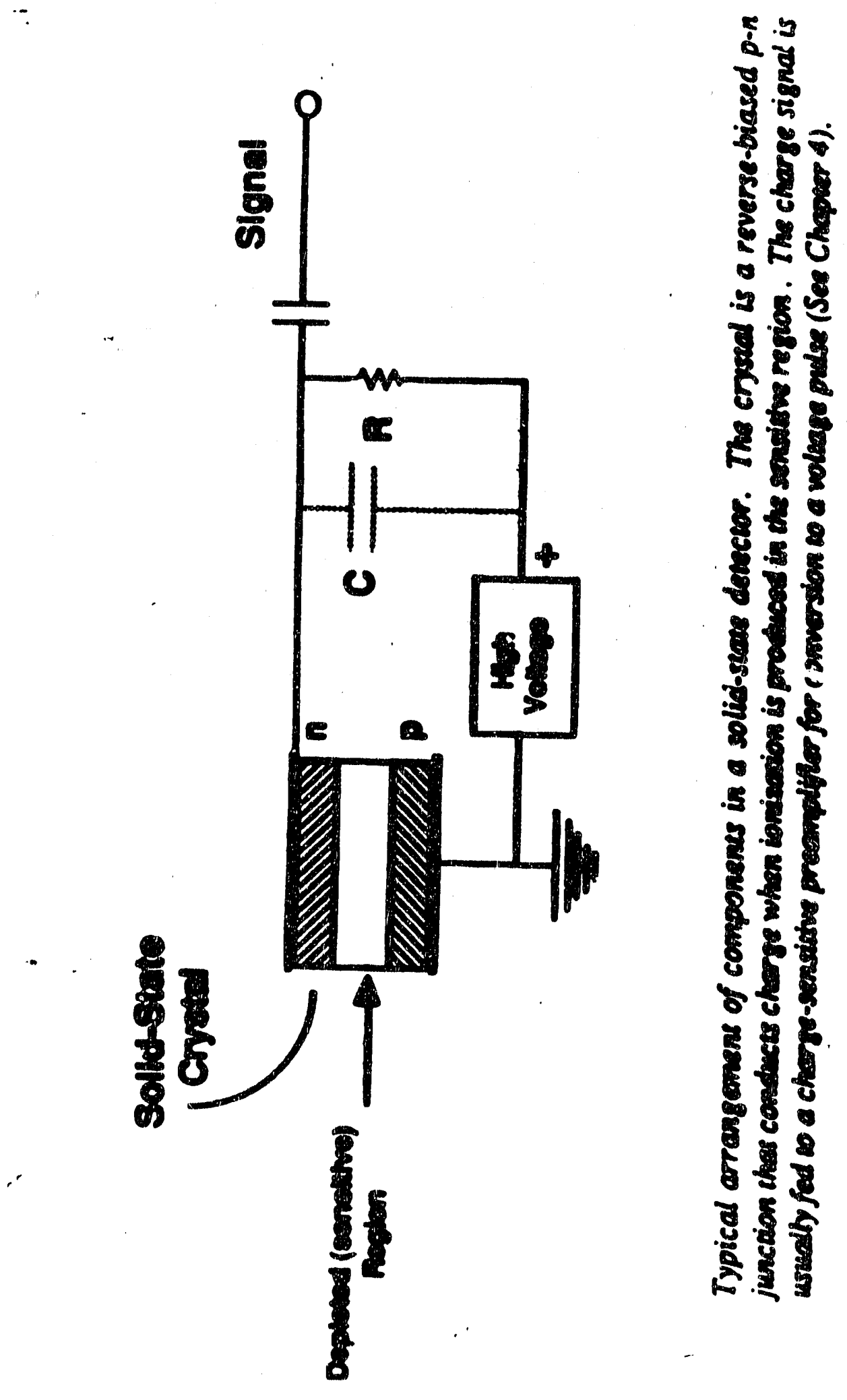




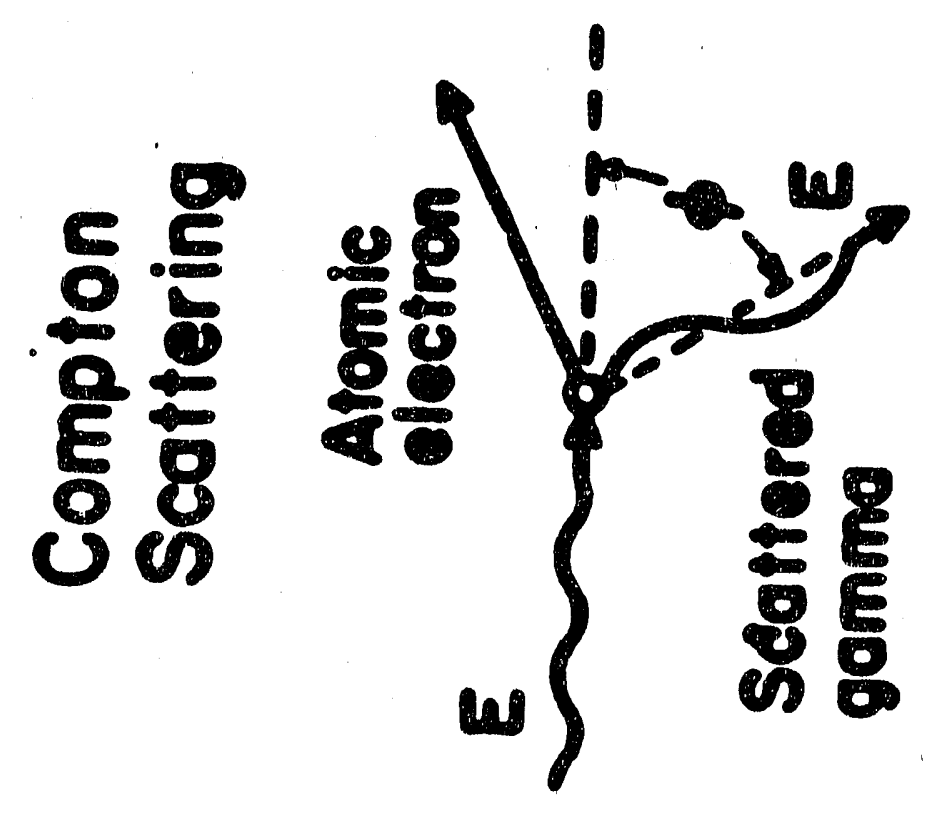



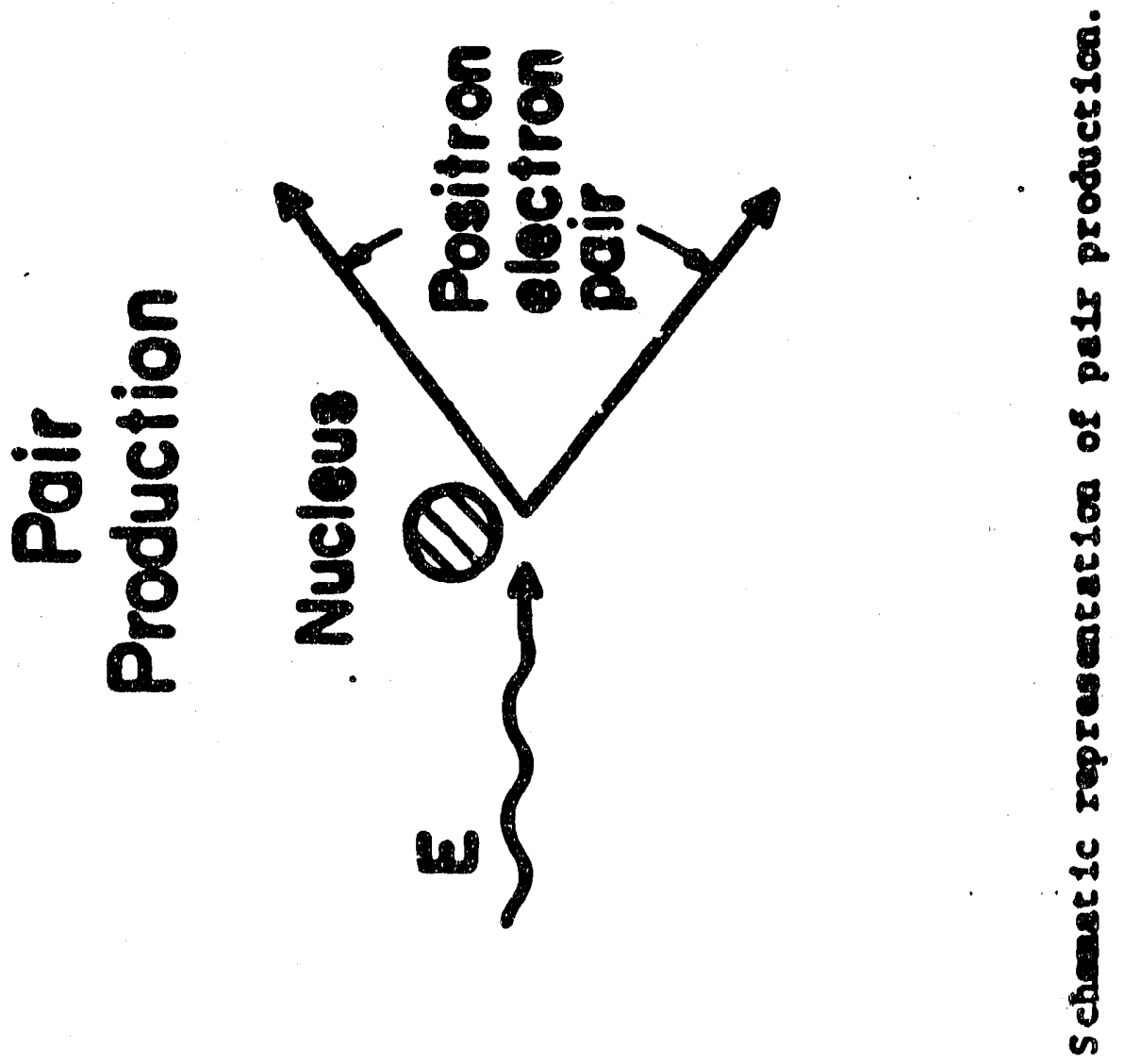


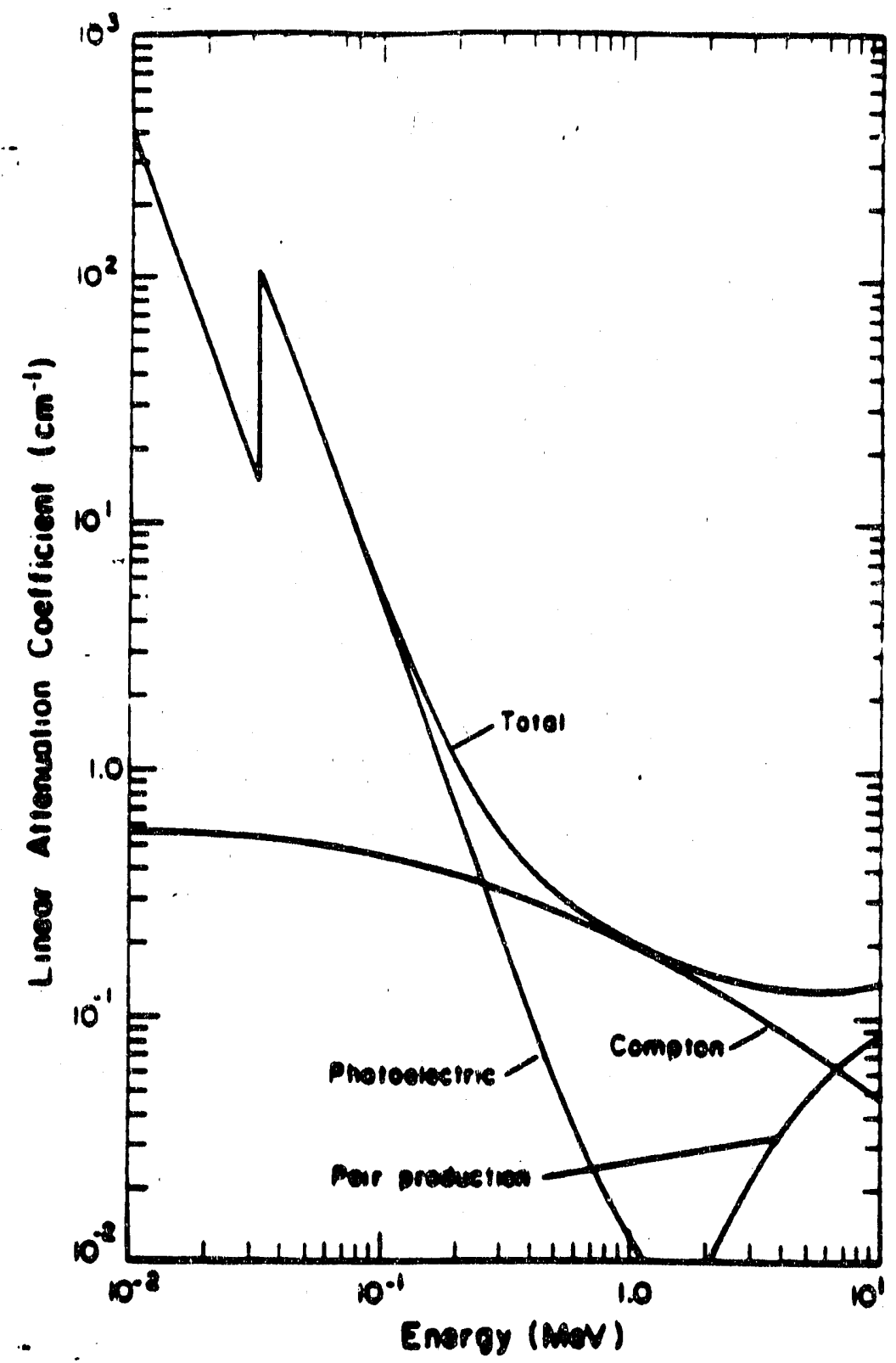

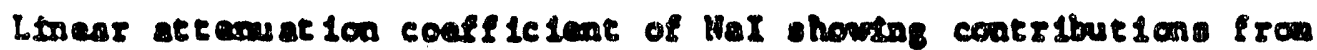
photoelectric abeorbeton, compton ecatertas. and pals production. 


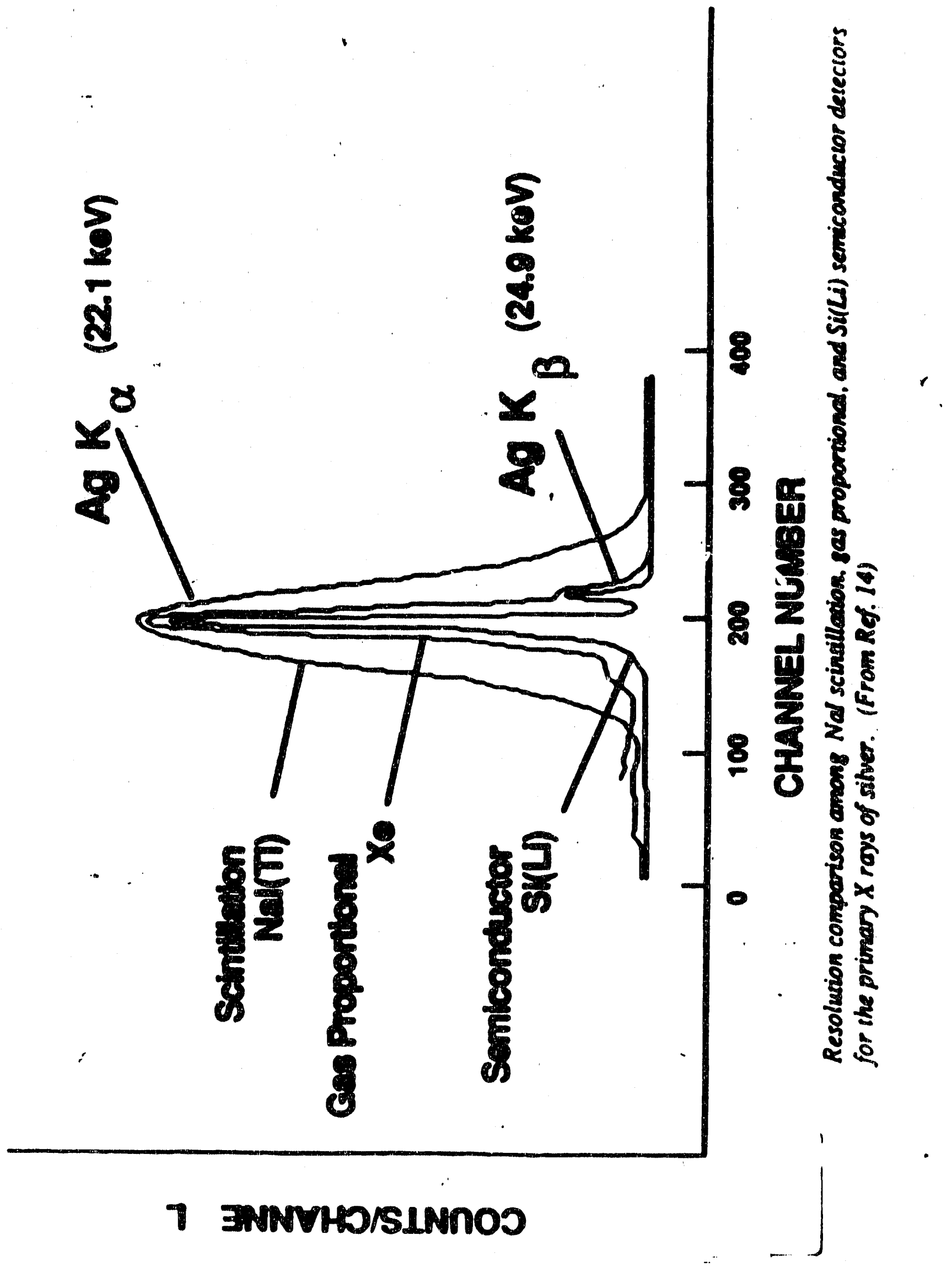




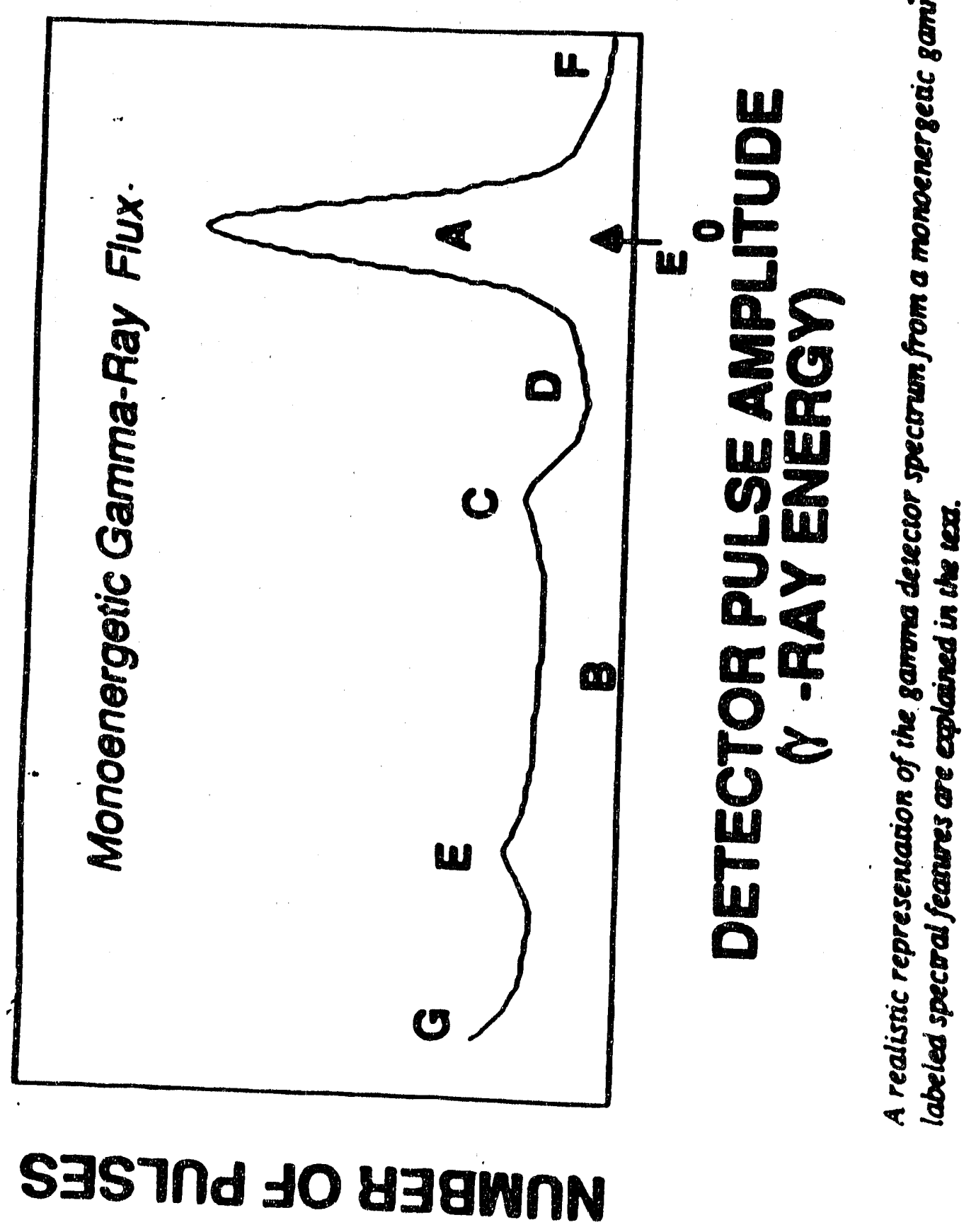




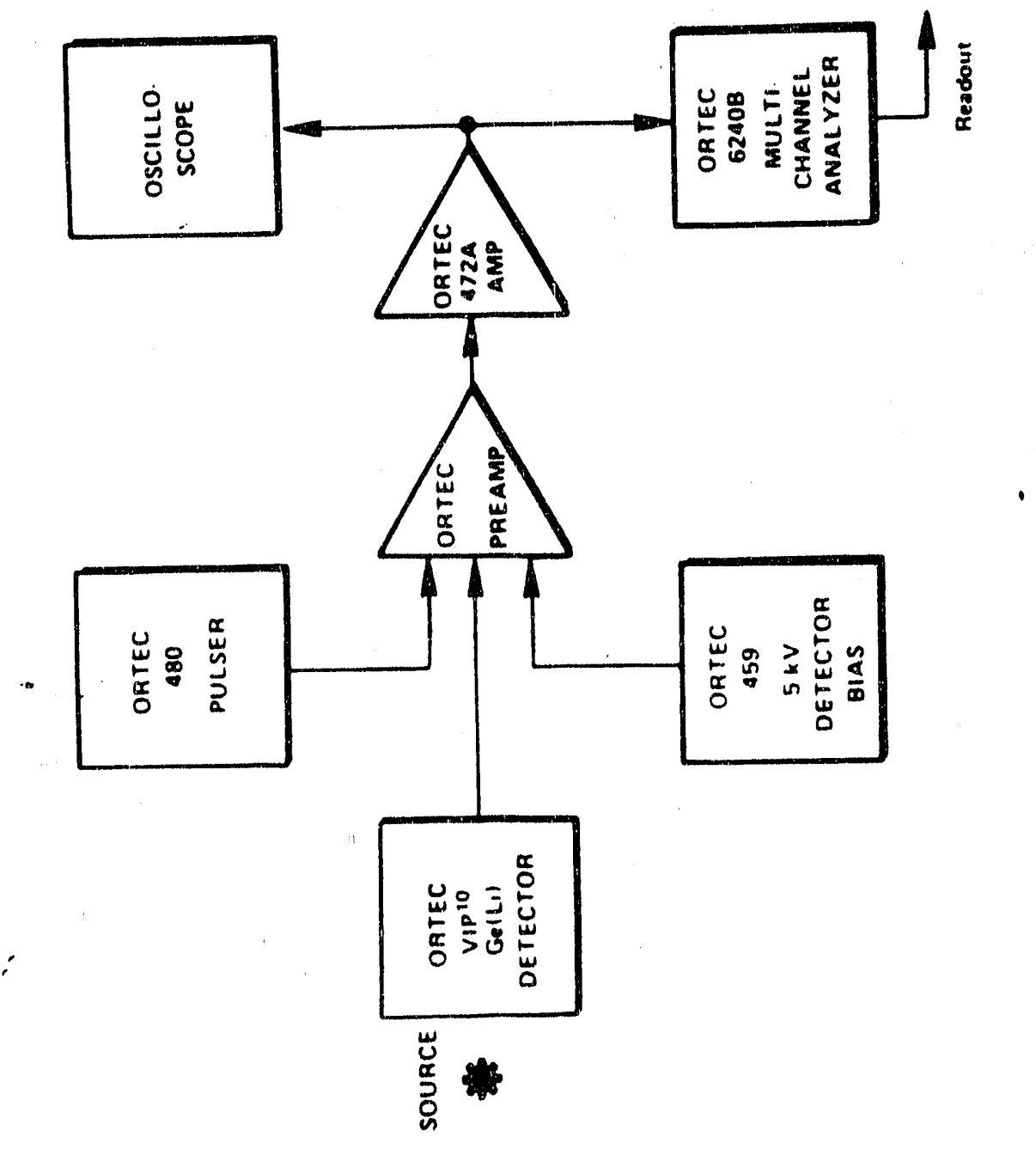




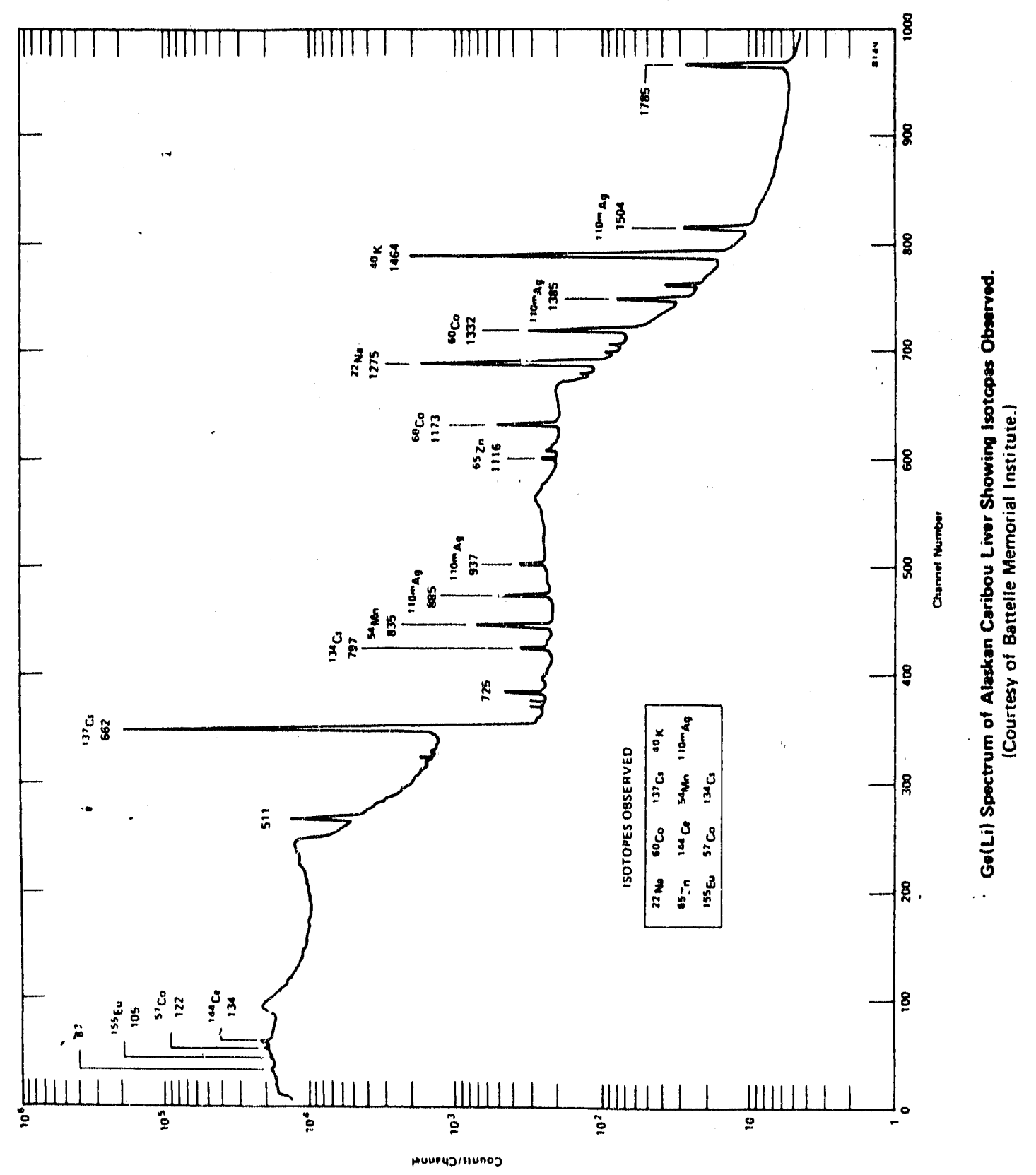




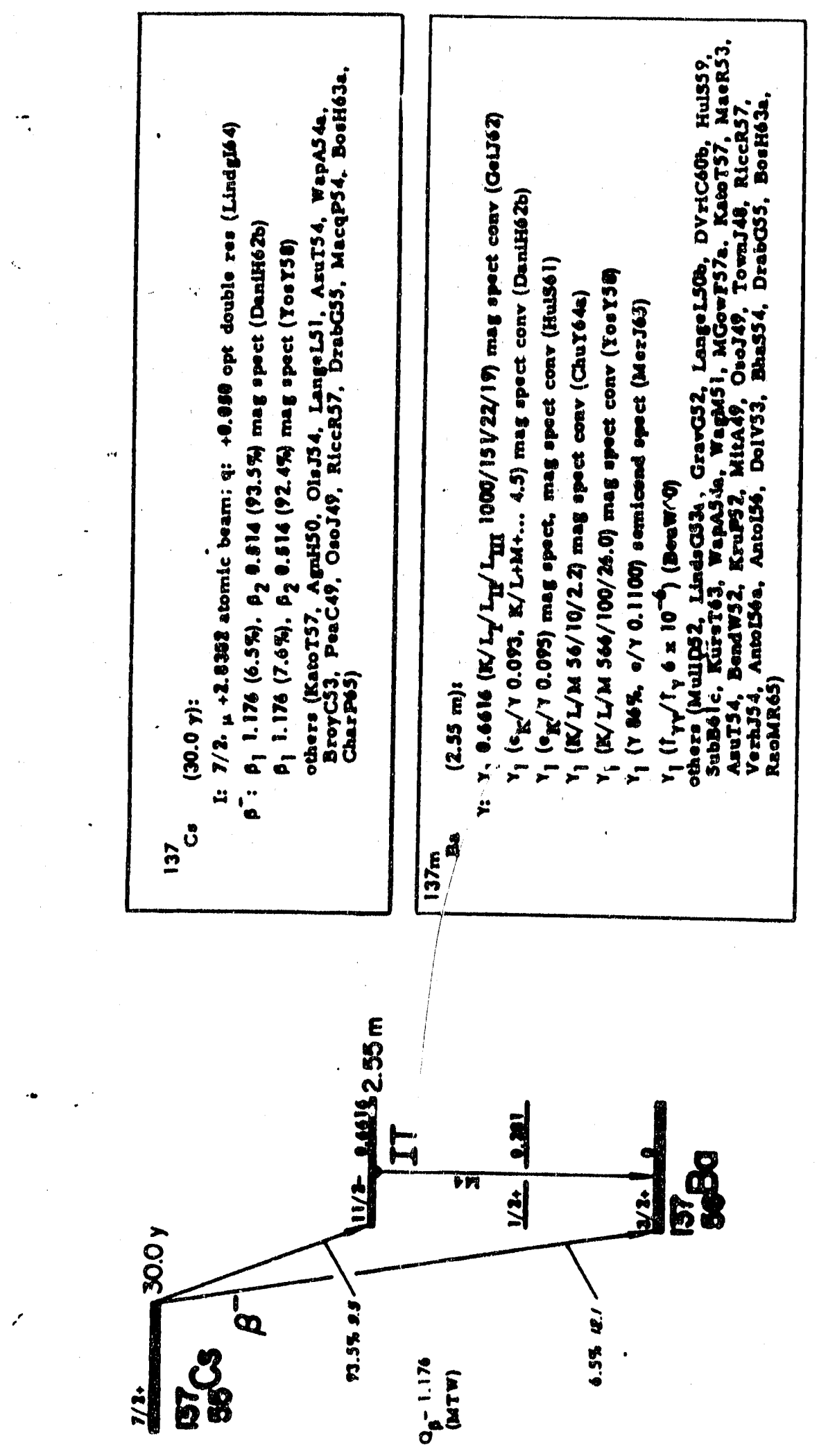




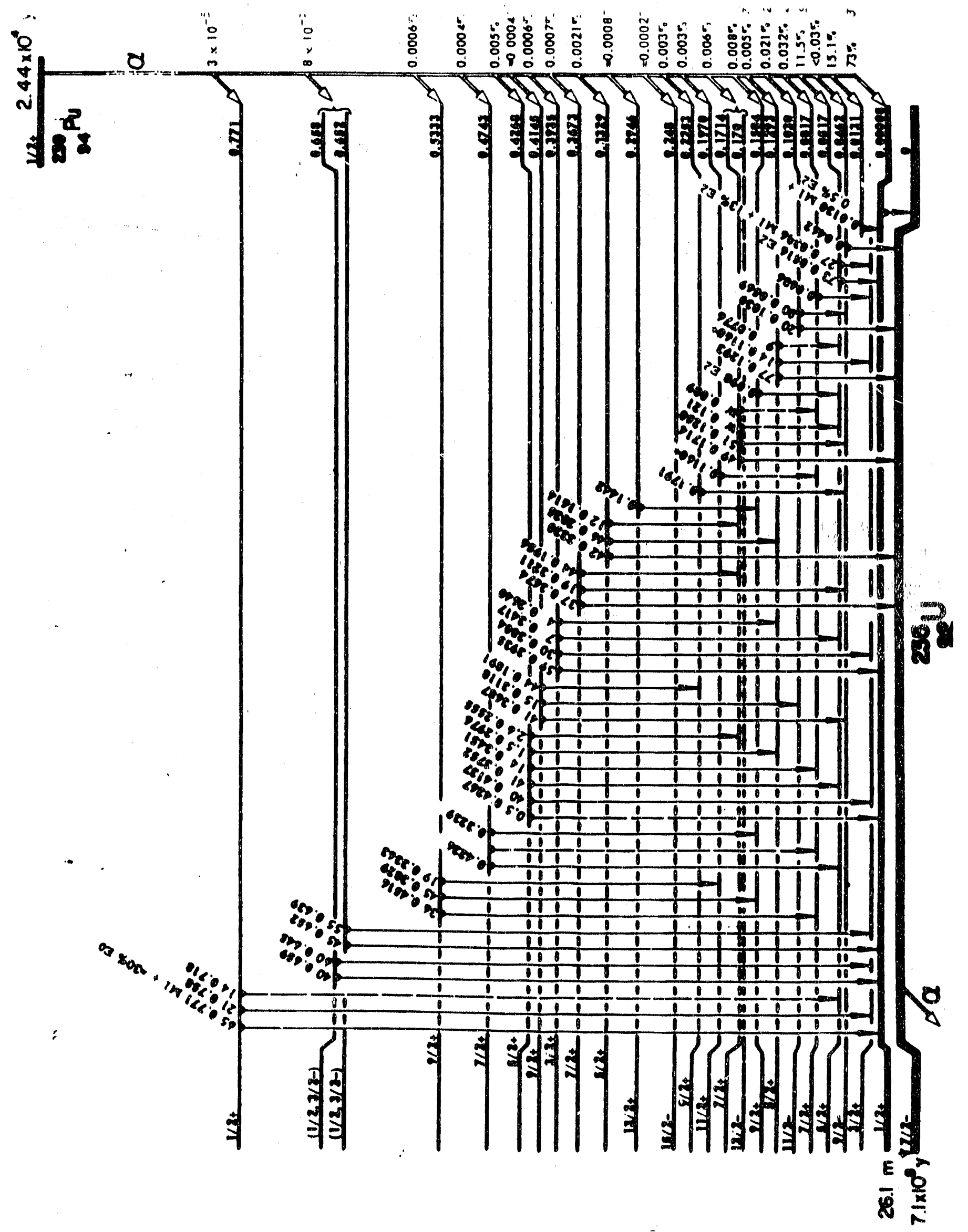




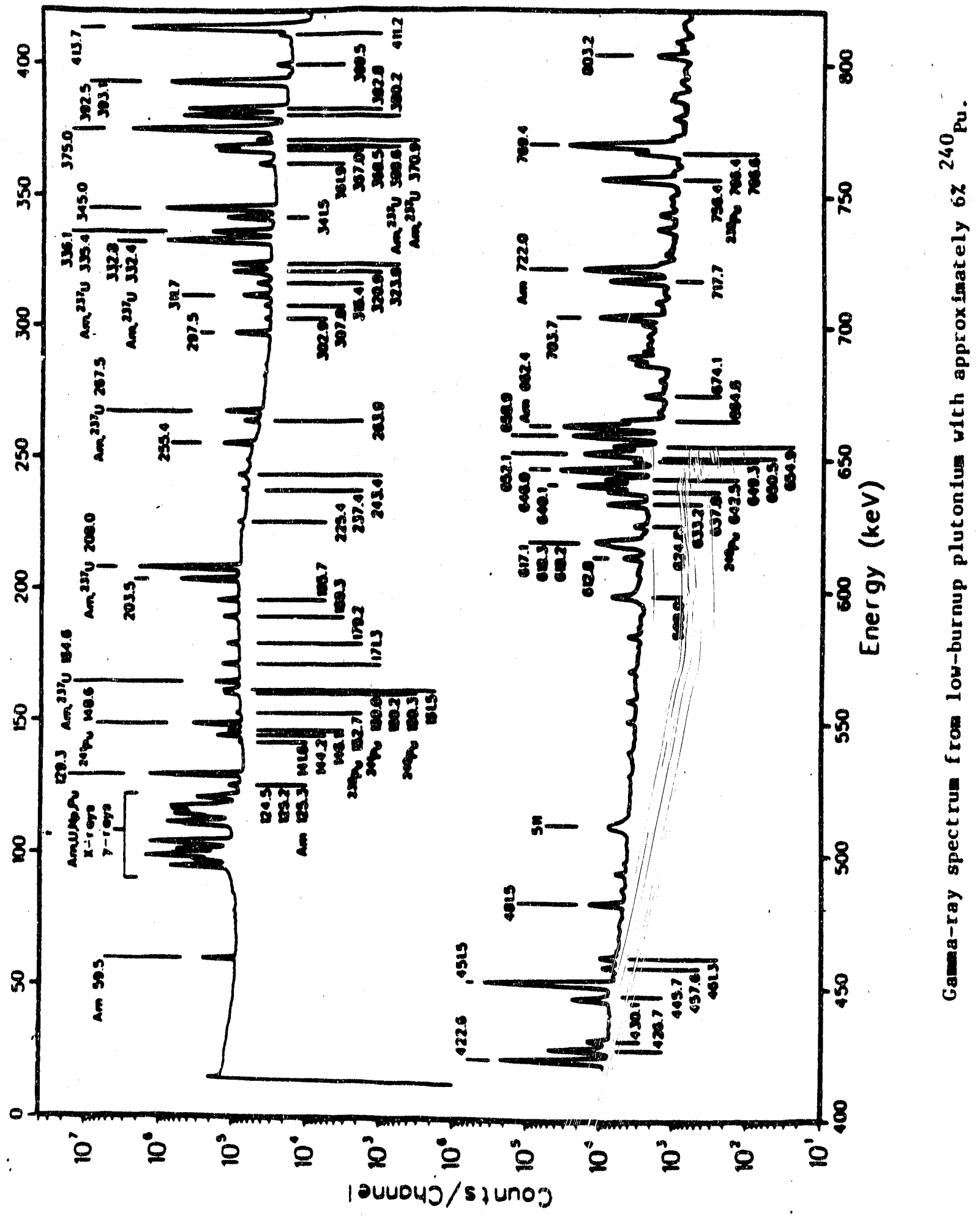




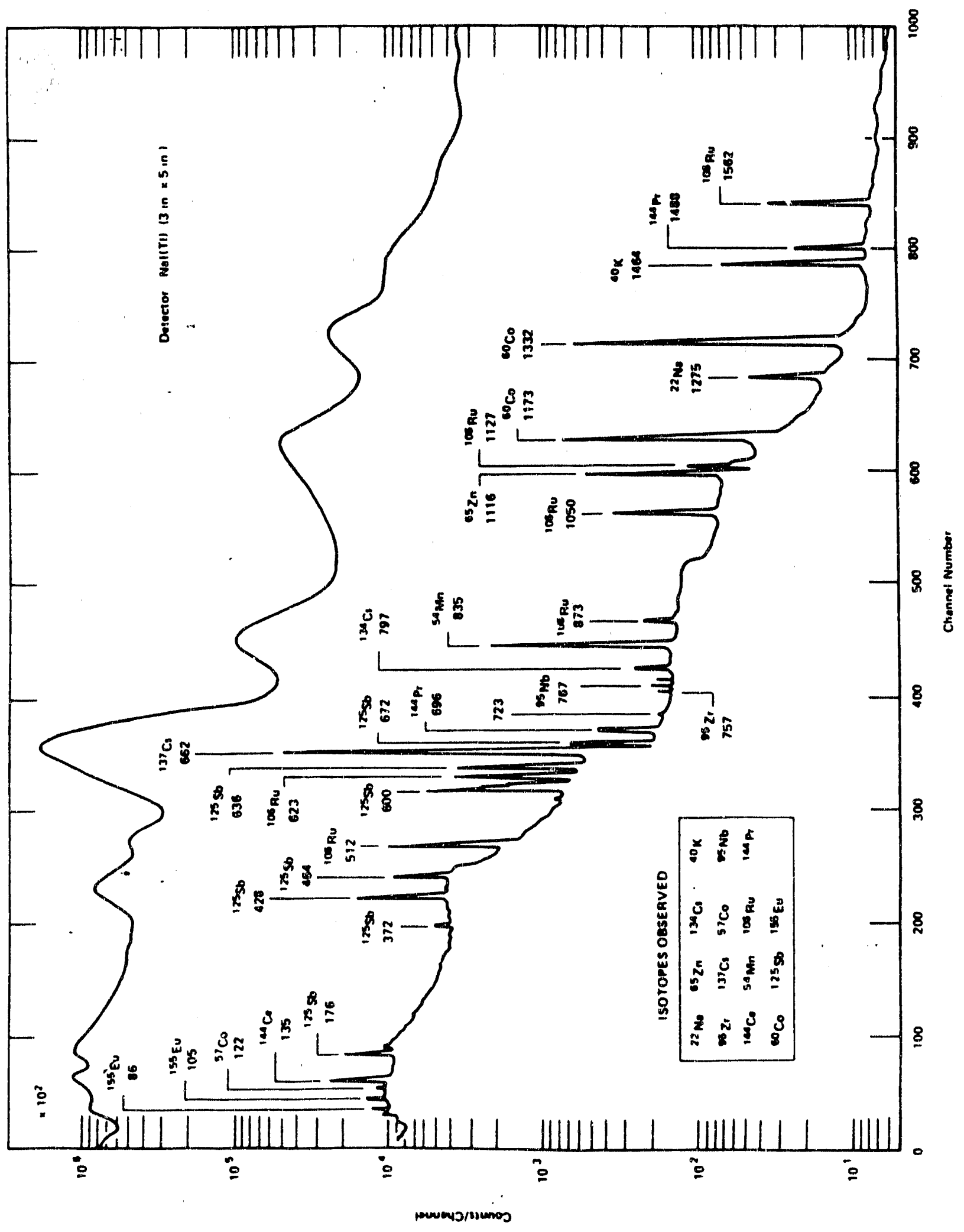




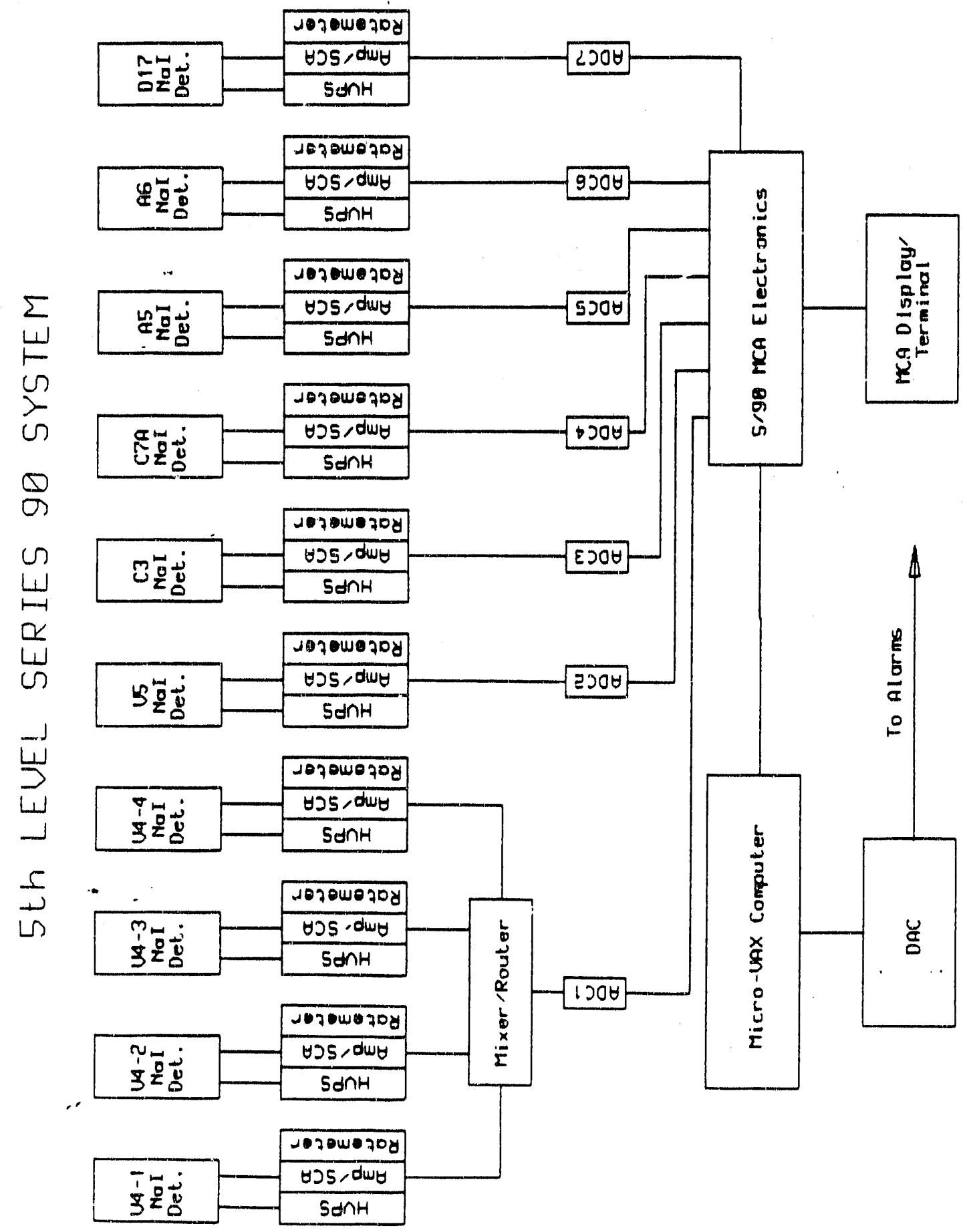


Assuming a half-life of $100 \mathrm{yr}$ and an atomic weight of 1.00, what weight of the radionuclide would give a rate of 6 $\mathrm{d} / \mathrm{m}$ ?

$$
\begin{aligned}
-d N / d t & =N \lambda \\
N & =(-d N / d t) / \lambda \\
\lambda & =0.693 / t_{1 / 2} \\
\lambda & =0.693 /(100 \times 365 \times 24 \times 60) \\
\lambda & =1.32 \times 10^{-8} \\
N & =6 / 1.32 \times 10^{-8} \\
N & =4.55 \times 10^{8} \\
W & =N \times M W / A \\
W & =\left(4.55 \times 10^{8} \times 100\right) / 6 \times 10^{23} \\
W & =7.6 \times 10^{-13} \mathrm{~g} \text { or } 1 \mathrm{~g} / \mathrm{million} \text { tons }
\end{aligned}
$$


X-Ray Fluorescence

K/L-Edge Densitometry

Neutron Activation

Cf Shuffler

Photo Dissociation 

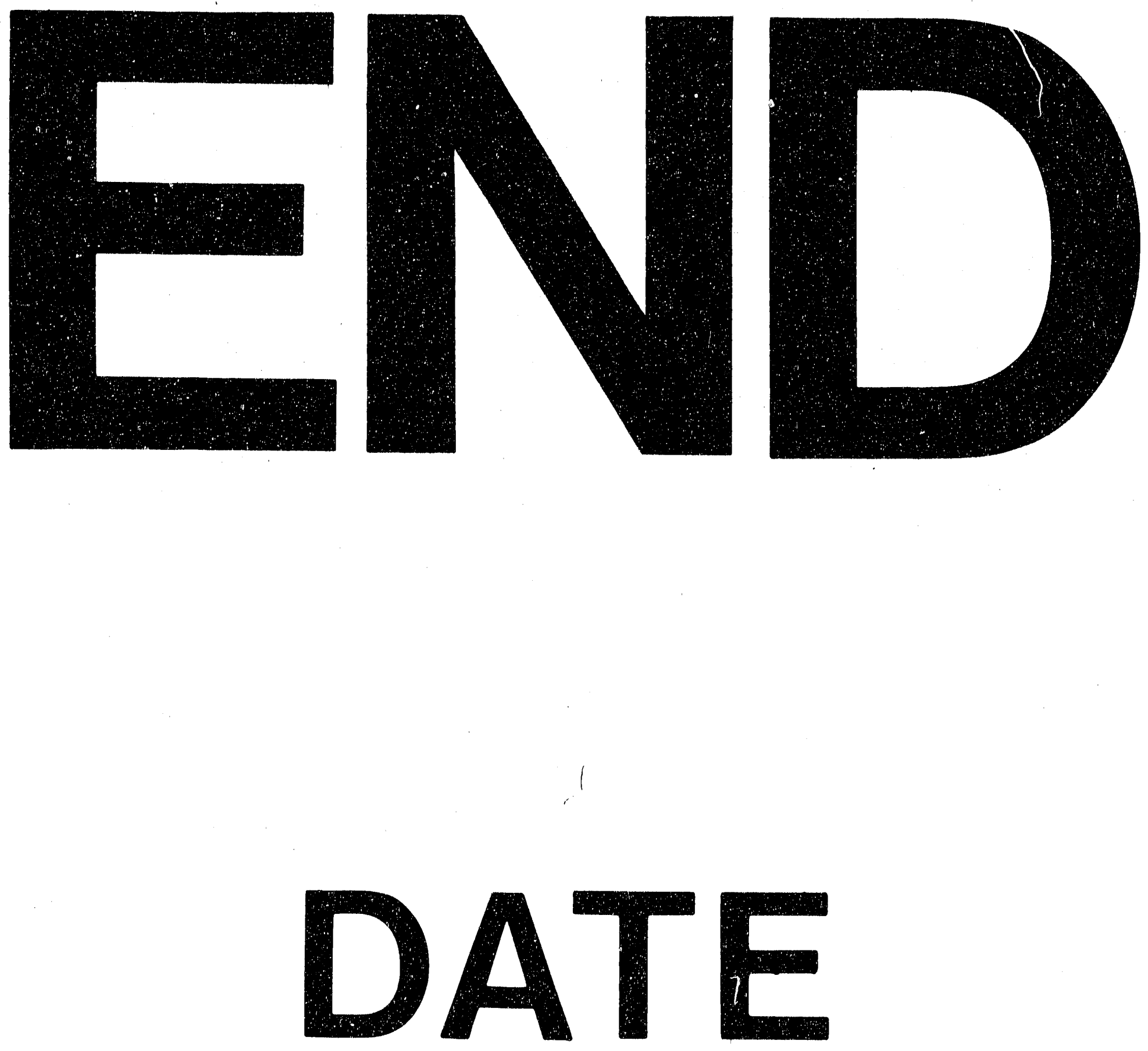

$\exists$
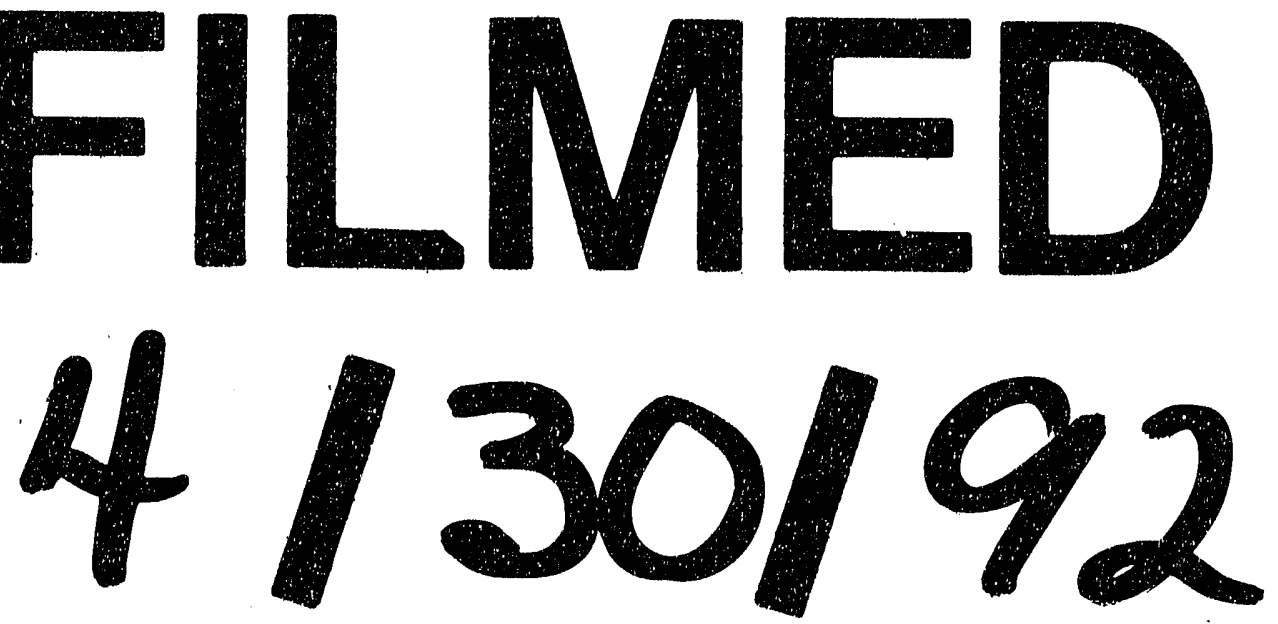
